\title{
PERSPECTIVAS DE HISTORIA Y CONTEXTO CULTURAL EN LA ENSEÑANZA DE LAS CIENCIAS: DISCUSIONES PARA LOS PROCESOS DE ENSEÑANZA Y APRENDIZAJE*
}

\section{Perspectives of history and cultural context in the teaching of Science: arguments for teaching and learning}

\author{
Fredy Ramon Garay Garay ${ }^{1}$
}

\begin{abstract}
Resumen: Este trabajo pretende socializar y retroalimentar determinadas reflexiones que surgen en torno de las relaciones existentes entre: Historia de las Ciencias, Contexto Cultural y Enseñanza de las Ciencias. Asumidas como perspectivas posibles, que poseen un alto potencial de transformación de los procesos de enseñanza y aprendizaje. Dichas reflexiones adquieren un carácter incluyente que no se limita a la formación de profesores sino que se extiende a la formación en ciencias en general. El trabajo se estructura en: 1. Historia de las ciencias; 2. Contexto cultural; 3. Enseñanza de las ciencias y 4. Relaciones existentes entre estas. No se asume que tales reflexiones sean la conclusión de dicho abordaje, muy por el contrario, se presenta este como una invitación al lector a pensar en torno de la enseñanza desde una perspectiva histórica y cultural y cómo este hace posible entender de una manera asertiva lo que puede significar educar en ciencias.
\end{abstract}

Palabras clave: Historia de las ciencias. Contexto cultural. Educación en ciencias.

\begin{abstract}
This paper attempts to socialize and provide feedback on certain thoughts that arise about the relationships between: the History of Science, and the Cultural Context of Science Teaching. Assumed as potential view points, which have a high potential for transforming teaching and learning. These reflections take on an inclusive character that is not limited to the training of teachers but extends to training in general in science. The work is divided into: 1. History of science, 2. Cultural context, 3. Science education and 4. Relationships between them. It does not assume that such reflections conclude such an approach, quite the contrary, it presents this as an invitation to the reader to think about teaching from a historical and cultural perspective and how this makes it possible to understand what this might mean for science education.
\end{abstract}

Keywords: History of Sciences. Cultural context. Science education.

\footnotetext{
*Una versión ampliada de este trabajo fue presentada como proyecto de pesquisa en el proceso de selección del Programa de Pós-graduação em Ensino, Filosofia e História das Ciências (nivel Doctorado), Universidade Federal da Bahia. Proyecto orientado por el profesor Charbel Niño El-Hani y coorientado por la profesora Maria da Conceição Marinho Oki.

${ }^{1}$ Licenciado em Química. Doutorando, Programa de Pós-graduação em Ensino, Filosofia e História das Ciências, Universidade Federal da Bahia. Salvador, BA, Brasil. <licfredygaray@yahoo.es>

Rua Goiás, Número 354, apto. 802

Bairro Pituba - Salvador, BA

41.830-130 


\section{Introducción}

El estudio de las posibles relaciones existentes entre historia de las ciencias, contexto cultural y enseñanza de las ciencias, emerge como propuesta inicial de investigación en el marco del proyecto de formación doctoral del autor. Esto se debe a que estas perspectivas contempla dimensiones de las ciencias como filosofía, sociología, antropología, historia y contexto socio-cultural, las cuales deben ser contempladas en los currículos, no solo como delineantes o condicionantes del diseño de éste, sino que por el contrario, dichas dimensiones deben ser visibilizadas en un qué hacer tanto de profesores como de estudiantes que se forman en ciencias.

La historia y la filosofía de las ciencias en los últimos años, ha sido un campo emergente, en el cual se han desarrollado un número significativo de investigaciones, que consolidan y estructuran la didáctica de las ciencias (MATTHEWS, 1994). Por su parte, el contexto cultural "la cultura", ha sido objeto de indagación de áreas como la sociología y la antropología de las ciencias. Este trabajo, pretende poner en evidencia las relaciones que existen entre la historia de las ciencias y el contexto cultural en la evolución del saber científico y de las prácticas científicas. Del mismo modo, establecer como estas dos dimensiones pueden impactar de forma positiva los procesos de enseñanza y aprendizaje de las ciencias, desde una perspectiva incluyente, es decir, que no solo se limite a la formación inicial y continua de profesores, sino también que involucre a otros profesionales, y así mismo, que sea el punto de partida, para posteriores investigaciones que den cuenta de estas dimensiones en los diferentes contextos de formación: educación básica, media, técnica y tecnológica, superior y post-graduación, que presenten en su currículo áreas de formación en ciencias.

Las investigaciones realizadas en torno a una educación en ciencias desde una perspectiva histórica, han dado resultados positivos en cuanto a los procesos de enseñanza y aprendizaje (EyA). Autores como Adúriz-Bravo (2000), Quintanilla (2005), Izquierdo (1994), Garay (2007), Martín del Pozo y Solsona (2004), entre otros, coinciden con el planteamiento anterior, sin embargo, se propone la necesidad de hacer de estas investigaciones, evidencias que posibiliten consolidar este campo de trabajo y que los resultados demanden cambios en lo metodológico, procedimental, conceptual, curricular y demás aspectos que son inherentes, dependientes de la formación en ciencias.

\section{De la historia de las Ciencias}

La historia de las ciencias, es un área de de investigación e indagación que emerge aproximadamente a mediados del siglo XX. Pero las investigaciones en torno a la incidencia de la historia de las ciencias sobre el proceso de EyA tiene como uno de sus posibles orígenes los trabajos desarrollado en la universidad de Harvard, referidos a estudios, por parte de los estudiantes de humanidades, de casos históricos basados en el análisis de procesos claves, en el desarrollo de la ciencia, con sus implicaciones filosóficas, sociales, culturales, políticas económicos entre otras (GARAY, 2007).

Si bien estas investigaciones han arrojado resultados que orientan el camino hacia nuevas metodologías y estrategias de trabajo al interior de las aulas de formación, hacia el diseño y reformulación de los currículos de ciencias, y marcan pasos que muestran un cierto 
sentido, sin afirmar que sea acertado o no, y que han ido delimitando un camino en tal dirección, no se debe dejar de lado, que tales investigaciones, también deben ser orientadas hacia la historia y el contexto cultural en sí. Esto es, tener claridad en torno a los objetos de investigación en historia de las ciencias y el contexto cultural, los enfoques bajo los cuales son desarrolladas estas investigaciones y las perspectivas y conclusiones de las mismas, que contribuyan de forma directa o indirecta sobre los procesos EyA.

Dentro de esta perspectiva de enseñanza de las ciencias, corresponde ahora presentar algunas reflexiones en torno a aquello que se ha denominado como historia de las ciencias. Si bien, la historia es un proceso constante de reconstrucción de hechos sucedidos en un periodo de tiempo único y específico, la ciencia no escapa a dichas formas de reconstrucción, ya que sean o no permeadas o mejor determinadas por estas, es la única vía que se tiene de conocer lo que sucedió en el pasado, en relación a lo que la comunidad de especialista a consensuado bajo el nombre de ciencia.

Una reconstrucción de la historia de las ciencias, debe contemplar diferentes aspectos que se pueden enmarcar en cuatro contextos que son: social, político, económico y cultural (GARAY; GALLEGO; PÉREZ, 2006), que dan cuenta de cómo ha sido la construcción y reconstrucción del conocimiento científico, de las practicas y de los instrumentos, además de los hombres y mujeres que se han dedicado a dichas prácticas científicas. Esto en pretensión de cumplir el objetivo de consolidar un carácter humanizado de las ciencias.

Un estudio de la historia de la ciencias desde un enfoque definido, pero ni único ni fijo, sino como lo plantea los expertos, desde los personajes (mujeres y hombres dedicados a las practicas científicas), los instrumentos (equipos, materiales, aparatos entre otros) los métodos (practicas, experimentos, técnicas entre otros) o los textos (escritos, grafos, dibujos, diseños y otros), como un proceso de reconstrucción cultural del saber científico, permite reelaborar la visión de ciencia y de la naturaleza de está. Esto es, mostrar la ciencia como una construcción no ajena a un cotidiano que interactúa constantemente con este, en un proceso dinámico y cíclico, en el sentido de que parte de una u otra y retorna, con modificaciones, reestructuraciones o nuevas demandas y necesidades. Proceso realizado por seres humanos, con habilidades, procesos, aptitudes y actitudes que les posibilitaron construir, reconstruir, formular o proponer posibles formas de ver, explicar y entender el mundo.

A su vez, los procesos de EyA desde un enfoque histórico, posibilitan una apropiación de conceptos, formas de significar y de sus formulaciones, debido a que desde esté, se hace posible identificar los aspectos que llevaron a su formulación y devela las transformaciones intra e interestructurales que han sufrido, el por qué han cambiado dependiendo de cada contexto, entendiendo la dinámica del saber científico, como una construcción y reconstrucción permanente de saberes, procedimientos, técnicas y demás actividades referidas a las practicas científicas.

La implementación de metodologías de enseñanza desde enfoque histórico, se han convertido en poderosas herramientas de apropiación y comprensión de modelos científicos (procesos de conceptualización), que permiten valorar y enseñar la ciencia dentro de diferentes espacios educativos, dicho de otra forma, poder hacer de los modelos científicos objeto de trabajo en el aula, bien sea, mediante los presupuestos teóricos que los didactólogos han denominado como modelación y donde establecieron diferentes categorías como modelos científico-escolares o modelos didácticos (GALAGOVSKI; ADÚRIZ-BRAVO, 2001), contextualizados en sistemas políticos, económicos, sociales y culturales específicos que permiten la configuración y consolidación de los modelos abstractos como constructos resultantes del 
proceso de EyA de las ciencias (GARAY, 2007). De forma contraria, investigaciones orientadas por este objetivo han obtenido como resultado que una enseñanza ahistórica presenta un visión de ciencia, reducida a sucesiones de hechos aislados unos de otros y completamente descontextualizados de un espacio y un tiempo determinado.

Una formación en ciencias desde una perspectiva histórica, permite generar una visión diferente de estas, ya que deja al descubierto los factores directos, indirectos precedentes e implicados en su surgimiento y posterior consolidación, esto como producto de los cambios en los contextos sociales, políticos, culturales y económicos. Además de permitir comprender que la ciencia tiene un pasado, el cual es consecuencia y a la vez fuente del presente -entendiendo lo anterior no como la mera reducción de la historia a una causa precursora, sino muy por el contrario como un espacio de indeterminadas posibilidades-, y que a su vez podrá o no influenciar el futuro, interviniendo o no con la construcción, predicción o modificación de éste ultimo. La historia al suministrar explicaciones coherentes, para los hechos que han originado los modelos científicos, al presentar interpretaciones rigurosas para los múltiples e intrincados procesos de conocimiento, contribuye eficazmente a la labor de desmitificación de las ciencias y generar espacios de reflexión y de proyección de las mismas.

La significación de la ciencia no se limita a la mera formulación matemática, a la memorización de signos y símbolos, sino que va más allá de la ductilidad y maleabilidad de palabras en busca de la formación de un pensamiento de carácter conceptual. El reconocimiento de la historia de los conceptos al igual que sus formas particulares de describir, explicar y predecir la aquello que denominamos como realidad, constituyen instrumentos muy valiosos para la docencia, posibilitando un proceso de EyA de las ciencias con mayores perspectivas y de más bastos alcances culturales, políticos, sociales y económicos.

La historia concebida como reconocimiento de modos de pensamiento y de maneras de ser, como esclarecimiento de las concepciones fundamentales que determinan la actividad científica, como identificación de los principios que en cada época organizaron y articularon las diferentes formas de ver o concebir la naturaleza, es indudablemente un elemento valioso en la formación científica, pues permite organizar las experiencias educativas de tal manera que satisfagan los requerimientos epistemológicos y psicológicos exigidos por la conceptualización científica.

Con este planteamiento se muestra que es posible una enseñanza de las ciencias a partir de un enfoque o perspectiva histórica, permitiendo contextualizar al estudiante en un marco social, político económico y cultural, que posibilita una versión diferente de la ciencia en lo conceptual y metodológico que le faculte para intervenir en el desarrollo de la misma desde un cambio actitudinal y axiológico.

\section{Contexto cultural}

La pretensión de definir la versión de contexto cultural en la que se inscribe este trabajo, conduce a intentar definir que es cultura y que es contexto, de esta forma, se encuentra que, como sucede actualmente con algunos términos científicos, ellos son o indefinibles o por el contrario son poli-definidos, y en este caso -cultura- no escapa a ello. Por tanto, es posible citar acá definiciones como las presentadas por Kroeber y Kluckhohon (1952), para quien, este termino presenta mas de una definición y ella depende de contextos determinados. En- 
tonces podemos entender cultura como: El modo de vida de un pueblo, Un legado social que el individuo adquiere de su grupo, Una manera de pensar, sentir y crear, Un depósito del saber almacenado, Conducta aprendida, Un mecanismo de regulación normativa de la conducta, Una serie de técnicas para adaptarse tanto al ambiente exterior, como a otros hombres, Un precipitado de la historia, Un tamiz, mapa, matriz. Estas definiciones son reforzados por Geertz (1987) quien establece que la cultura puede ser asumida como dos ideas básicas: una como realidad mental y la otra como contexto.

Uno de los obstáculos que se enfrenta, cuando se inicia una investigación en este campo, es el relacionado a intentar definir un concepto u otro, en este caso cultura o contexto, y en este sentido, contexto se define o mejor el sentido de su forma de significar se podría resumir groseramente como un ente imaginario que delimita alguna cosa, esto es, una línea imaginaria o mejor un recipiente imaginario en el cual es posible depositar absolutamente todo, e este recipiente permite relaciones entre los diferentes elementos contenidos, así como con los elementos de otros recipientes lo que ha sido denominado por algunos investigadores como cruce de frontera cultural. Podría establecerse como un lugar de contacto en donde se comparte la cultura, donde tan contacto se encuentra mediado por tradiciones, símbolos, signos e toda posible forma de comunicación.

Esta definición de contexto presenta un carácter dinámico tal como lo presenta Young (1999) sin embargo, ya la cultura, es definida desde una perspectiva mas lingüística. Según esta perspectiva contexto y cultura no son homogéneos, ya que, ellas difieren en varios aspectos, entre ellos los posibles límites, y son estos precisamente, los que pueden llegado el caso, influir directamente en la historia y en la educación en ciencias.

De acuerdo con lo anterior, Forquín (1993) propone una explicación que permite ir entretejiendo la relación entre contexto y cultura, para poder marcar un horizonte en búsqueda de definir lo que sería contexto cultural, en particular él considera que la selección cultural depende de las relaciones entre Conocimiento y Cultura que cada proyecto educativo establezca. La selección al interior de una cultura, posee una doble importancia: con ella se realiza una reelaboración de los contenidos culturales que se transmiten a las nuevas generaciones. No se transmite la cultura como un repertorio simbólico unitario, tal transmisión también está sujeta a los azares de las relaciones simbólicas y los conflictos de interpretación.

Diferentes investigaciones en sociología y antropología de las ciencias, señalan que esta dinámica de selección cultural no termina en el currículo formalizado, sino que ella es realizada por la enseñanza; un ejemplo podría ser observado en varias investigaciones en el campo de la educación en ciencias. Es entonces, visible que la relación de la cultura con la historia y la educación va más allá de la simple formulación de un currículo, sino que presenta una seria de aspectos relevantes a ser considerados.

Con un enfoque histórico-crítico-cultural de la enseñanza de las ciencias, Mathy (apud ANDRADE, 2000), destaca la constitución de epistemes, en torno a teorías y conceptos específicos, asociadas con aspectos histórico-culturales. En un análisis sobre los textos escolares, específicamente en el tratamiento de las teorías de la evolución de la vida en el contexto francés, establece una rejilla de análisis que involucra la crítica epistemológica y establece tendencias tanto constructivistas como positivistas del concepto, así como aspectos ideológicos y culturales. Así, el concepto de evolución de la vida se entiende como una especialización, en la disciplina de la biología, de una visión más amplia de la idea de progreso subyacente al desarrollo de la naciente sociedad europea 


\section{Enseñanza de las Ciencias}

Con los cambios que se suceden en el interior de la ciencia y la tecnología, se hace necesario un cambio en la educación que reoriente la enseñanza y el aprendizaje de las ciencias. Este cuestionamiento lo deja entrever Ratke, profesor Alemán, entre 1614 y 1618, quien preocupado por el mejoramiento de las estrategias de enseñanza se atreve a hablar de lo que él denomino "didácticus", pero es Comenio (1592-1670), quien reestructura las ideas de Ratke en su Didáctica Magna, estableciendo que la didáctica es el arte de enseñar todo a todos sin pérdida de tiempo.

Esta primera versión de didáctica nace en el interior de un contexto de industrialización que pretende la formación de mano de obra, asumiéndose la enseñanza como la transmisión y posterior repetición de contenidos, definiciones y formulaciones matemáticas, sobre la base de la construcción y reconstrucción de conductas deseables y la eliminación de todas aquellas que no se ajustaran a lo buscado.

Es a partir de estos presupuestos, que el proceso de EyA adquiere un carácter operacional mecánico, con la posterior producción lineal de operarios con conocimientos memorísticos de tipo mecanicista, en donde solo es necesaria la transmisión de contenidos para ser aprendidos y con la posterior repetición de estos para ser aplicables dentro de un contexto de producción efectiva.

Dentro de este ámbito de industrialización, se hace evidente la analogía del profesor como tecnólogo, quien es preparado para cumplir con un oficio, durante lo que se considera su práctica docente, limitándose a la transmisión-repetición de contenidos curriculares, convirtiendo el proceso de EyA de las ciencias en algo mecánico, estático, limitando el conocimiento a la memorización de conceptos sin significado o con poca repercusión en su significar, y a la matematización formal de los mismos.

A causa de esto, se hace necesaria la revisión y posterior reestructuración de esta didáctica, surgiendo así, lo que en la actualidad se conoce como la nueva didáctica de las ciencias, que dará origen a la didáctica específica de las ciencias de la naturaleza. Investigadores han planteando que el proceso EyA de las ciencias no debe estar viciado por la observación e imitación de otros.

Para lograr este cometido, se parte de la revisión de la perspectiva epistemológica que ha orientado dicho proceso y se llega a la conclusión de que ha sido el inductivismo el que ha direccionado la enseñanza y el aprendizaje de las ciencias. Por tanto, se debe dar inicio a esta reestructuración de la didáctica desde una nueva versión epistemológica, esta reestructuración se basará en los presupuestos teóricos del deductivismo y es configurada por los resultados de los trabajos realizados por Piaget, Ausubel, y Glasersfeld, quienes enmarcan una nueva corriente denominada constructivismo, dejando de lado la didáctica operacional, mecánica y estática, para dar paso a la didáctica desde el constructivismo moderado.

En esta nueva dirección, se asume el proceso de EyA de las ciencias, como objeto de investigación, permitiendo alejarse de las concepciones tradicionales, dándose así, inicio a investigaciones en este campo que le permitan consolidarse como una ciencia y tomar distancia de la didáctica general. Un primer acercamiento a la nueva didáctica de las ciencias se hace desde la versión epistemológica de S. Toulmin (1972), entendiendo esta, como una empresa racional en la que los profesores en ejercicio continuaban siendo considerados como aplacadores, dentro de los sistemas aula, de los desarrollos hechos por los investigadores. 
Es a comienzos de la década de los noventa que se hace posible empezar a considerar a la nueva didáctica como un cuerpo de conocimientos específicos. Por tanto, que su fundamentación teórica (conceptual y metodológica) es deber solo de una comunidad de especialistas que pretenden hacer de los modelos o teorías científicas objetos de trabajo en el aula. Ahora bien, la consolidación de la didáctica de las ciencias, ha permitido a los especialistas separarse de la práctica y tomarla como un objeto de reflexión, siendo esto observable en las diferentes etapas por las cuales dicha consolidación ha cruzado, y que ha dejado entrever que no es solo objeto de trabajo el saber sino también del saber hacer.

En el interior de las investigaciones que pretenden dar cuenta del carácter de cientificidad de la didáctica, han concluido por parte de los especialistas, que esta puede ser considerada como una tecnociencia, o ciencia de diseño, tendiente a mejorar el proceso de EyA de las ciencias, donde este sistema se compone de unidades fundamentales, que son los alumnos, los profesores, los contenidos curriculares y el contexto socio-cultural.

Investigadores en didáctica de las ciencias, consideran que el núcleo teórico de esta la didáctica- está constituido por la transposición didáctica (BACHELARD, 1985), que permite la recontextualización del desarrollo de teorías o modelos científicos. Esto es, una enseñanza significativa diferente al conocido proceso de EyA transmisión-repetición, la concepción de la evaluación como un instrumento que permita guiar el camino de la enseñanza y todo esto mediado por un leguaje científico.

Lo anterior establece a la didáctica como ciencia teóricamente fundamentada con campos específicos de investigación, enmarcados dentro de una corriente constructivista, (no siendo la única ya que actualmente podemos tomar este trabajo desde una versión mas sociointeraccionista), que le permiten hacer del proceso de EyA de las ciencias experimentales objeto de trabajo. Dentro de la nueva didáctica de las ciencias experimentales, la historia y la filosofía han adquirido gran relevancia, esto a causa de que han permitido a los profesores de ciencias explicar, comunicar y estructurar sus ideas acerca de la naturaleza de las ciencias, posibilitando conocer la ciencia que se enseña y facilitando el cómo enseñar.

\section{Relación entre historia de las Ciencias, contexto cultural y enseñanza de las Ciencias}

Las relaciones entre la historia de las ciencias y el contexto cultural, no son, como se pudiera considerar, lasos que emergen de la casualidad o el azar, sino que se pondría establecer una relación bidireccional en términos de interdependencia, es decir, tanto la una depende la otra y lo que se suceda en el interior de una se vera de una u otra forma reflejado en el carácter externo e interno de la otra. Es decir, todo aquello que influencia directamente en la historia tendrá un devenir contextual y viceversa. Esto es, en el caso de la ciencia, suponer que ella es una construcción resultante de las demandas y necesidades de las sociedades. Hecho enmarcado dentro de los contextos históricos y culturales que rodearon cada uno de los modelos científicos, que se han configurado en lo que conocemos, entendemos, estudiamos, analizamos, explicamos y concebimos como ciencia.

Para acompañar este proceso de reflexión, se presenta un caso particular que envuelve las tres dimensiones presentadas arriba, además de mostrar las posibles relaciones entre 
ellas. Este ejemplo permitirá desarrollar con mas precisión los objetivos planteados y el por qué de reflexionar sobre tales dimensiones.

\section{El caso de la formulación de la ley periódica}

Es importante reconocer que la ley y la tabla periódica de Mendeléiev formó parte de su libro de texto The Principles of Chemistry, (los Principios de Química, escrito entre $1868 \mathrm{y}$ 1870), el cual es escrito por D. Medeléiev, con la única pretensión de mejorar la compresión de esta ciencia por parte de sus estudiantes, así como de métodos de observación, hechos experimentales, las leyes de química, y el comportamiento en general de la materia. No obstante, cabe mencionar que el libro era claramente de química general.

El libro de texto de Mendeléiev (1869) pasó por ocho ediciones en ruso en su vida y cinco más después de su muerte. A pesar de tener mas de un siglo de la formulación de la ley y la tabla periódica y de su relevancia para la química y el proceso EyA de la misma, historiadores y filósofos de ciencia están intentando todavía entender su origen, naturaleza, evolucion e importancia en el desarrollo de la química como ciencia (SCERRI, 2007; BENSUIT-VICENT, 1991).

La mayoría de los historiadores considera que el congreso internacional celebrado en la ciudad Alemana de Karlsruhe entre el 3 y el 5 de Septiembre de 1860, fue un hito en la historia y en el desarrollo de la química. Un volante fechado el 10 de julio de 1860, fue enviado por los organizadores del congreso a la mayoría de los químicos excelentes de Europa y perfiló su objetivo como la necesidad de alcanzar un acuerdo general, pretendiendo hacer las definiciones más precisas de los conceptos de átomo, molécula, el equivalente, atomicidad, alcalinidad, entre otros, la discusión en relación con los verdaderos equivalentes de las sustancias y sus fórmulas; y la iniciación de un plan para una nomenclatura sistemática e única.

Mendeléiev, que para la época tenía la edad de 26 años, asistió al congreso y se impresionó grandemente por la contribución del químico S. Cannizaro, quien también participaba de tal congreso. En una carta del 7 de septiembre de 1860, resumió un logro importante de este congreso: Él decide entender de una forma diferente los conceptos de átomos y moléculas, asumiendo que una molécula era la cantidad de sustancia que entraba en una reacción y determinaba las propiedades físicas de los compuestos, mientras que un átomo era la cantidad más pequeña de una sustancia incluida en una molécula, pero D. Mendeléiev, va mas allá, y pretendió alcanzar la comprensión del concepto de equivalente, entendiéndolo como netamente empírico mientras no dependiera de los átomos o moléculas (GARAY, 2007)

Según el desarrollo histórico de la ley y la tabla periódica, contribuciones hechas por otras científicos como Dumas (1828 apud GARAY, 2007) Dobererienerg (1817 apud GARAY, 2007), Chancoutuois (1866 apud GARAY, 2007) y Meyer (1869 apud GARAY, 2007) entre otros, formulando sus propios modelos de tabla periódica, marcaron un hito en el desarrollo de la química como ciencia, y sus trabajos se convirtieron en algunos casos, en los presupuestos teóricos y experimentales de la propuesta de Mendeléiev, ya que particularmente en el caso de los trabajos de Meyer, este formuló la ley periódica en la misma época, sino que el enfoque de su trabajo fue diferente (GARAY, 2007).

En el trabajo que fue publicado por Mendeléiev el 17 de febrero de 1869, titulado Experimento de un Sistema de Elementos Basado en su Peso Atómico y en la Afinidad Química, presentó 
Perspectivas de historia y contexto cultural ...

su primer modelo de tabla periódica; además de formular lo que hoy se conoce como ley periódica estructurada a partir del convencimiento de que "las propiedades de cuerpos simples, la constitución de sus compuestos, así como las propiedades de éstos, son funciones periódicas de los pesos atómicos de elementos" (MENDELÉIEV, 1869 apud Garay, 2007, p. 69; RODRÍGUEZ et al., 2005, p. 86 ). En este trabajo, es en el cual Mendeléiev (1869) publico las siguientes conclusiones que constituían y estructuraban la formulación de la ley de periodicidad:

. Si los elementos se distribuyen de acuerdo con el aumento progresivo de sus pesos atómicos, se manifiesta una evidente periodicidad en sus propiedades.

. Los elementos con las propiedades químicas similares pueden tener pesos atómicos muy semejantes, como ocurre con el osmio, iridio y platino; o sus pesos atómicos aumentan progresivamente según una razón aritmética, como el caso del potasio, rubidio y cesio.

. La distribución de los elementos en grupos, según el orden de sus pesos atómicos, corresponde a un agrupamiento según el orden de sus valencias.

. Los elementos que están ampliamente difundidos en la naturaleza se caracterizan por sus pesos atómicos bajos y tienen propiedades marcadamente definidas. Estos son, por consiguiente, elementos típicos.

. El carácter de un elemento está determinado por la magnitud del peso atómico.

. Es de esperar el descubrimiento de elementos que aun no se conocen.

- Por ejemplo, los elementos análogos al aluminio y al silicio, cuyos respectivos pesos atómicos tendrán valores cercanos a 65 y 75 .

. El peso atómico de un elemento puede corregirse con la ayuda del conocimiento que se posea de los pesos atómicos de los elementos contiguos, así, por ejemplo, el peso de combinación del Telurio debe estar entre 122 y 126, no pudiendo ser 128.

. Es posible predecir ciertas propiedades de los elementos si se conocen sus pesos atómicos.

Con respecto al concepto de peso atómico, es importante la elucidación que hizo Stanislao Cannizaro (1860 apud GARAY, 2007) en el congreso de Karlsruhe, ya que fue crucial para la formulación del modelo de periodicidad. Curiosamente tres de los investigadores, que formularían el modelo de periodicidad química, estuvieron presentes en dicho congreso. Pero la elaboración de un modelo de tabla periódica era difícil, y tomó largo tiempo, debido a la falta de una concepción definida de peso atómico que está muy ligada con las definiciones de moléculas y átomos.

La disponibilidad de los pesos atómicos de aproximadamente 50 elementos le permitió a Mendeléiev (1869) ordenar los elementos en su Tabla, según las propiedades físicas y químicas como la densidad, el calor específico, el peso atómico, el volumen atómico, el punto fundición, la valencia, la formación de óxidos, cloruros, y sulfuros.

En contraste con otros formuladotes del modelo de periodicidad química, el trabajo de Mendeléiev (1869) se caracterizó por: la división en un grupo principal y subgrupos, dejando espacios libres para los elementos no descubiertos junto con la predicción de algunas de sus propiedades, como por ejemplo, la inclusión de algunos elementos homólogos, la posterior clasificación de los metales de transición, y la inversión de telurio-yodo.

El origen y posterior desarrollo del modelo de periodicidad química es atribuido a las disertaciones hechas por Cannizaro (1860 apud GARAY, 2007) durante el congreso de Karl- 
sruhe, donde hizo la distinción entre átomo, molécula y otros conceptos como valencia, que dieron origen y posterior desarrollo del modelo de periodicidad, pero no es posible dejar de lado la importancia que tuvo en este desarrollo el modelo atómico, encontrando en la historia que se le atribuye el éxito de la tabla periódica principalmente a las propiedades empíricamente observadas.

Es por tanto, que esta formulación ha sido de gran importancia para la historia y la filosofía de la química. Tal sistema periódico de los elementos formulado por Medeléiev, quien también lo reestructurará y perfeccionará, ha sido considerado como uno de los mas importantes avances al interior de esta ciencia. Y como una de las refleciones presentadas en este trabajo no es posible dejar de mencionar el hecho de su contribución a las discusiones de carácter epistemológico, ya que por primera vez en la historia de la química, se construye una organización del saber científico con un alto potencial predictivo en torno al comportamiento y las propiedades de los elementos. Se podría afirma entonces que la contribución de Mendeleev (1869), contribuyo para el estatuto de cientificidad de la química, con la sistematización de esta, partiendo de cuestionamiento que tenían que ver con su enseñanza y aprendizaje.

Con el ejemplo arriba presentado, se ha intentado caracterizar, algunas de las posibles relaciones existentes entre el la historia de la ciencias, el contexto cultural y la enseñanza de las ciencias. Sin embargo, es de mencionar que ciertas de las diferentes interpretaciones que se han hecho de la enseñanza de las ciencias desde la historia, han permitido formular diversas hipótesis que son tendientes a la formulación de currículos que presentan una versión deformada de esta (ahistórica), que se limita a citar hechos concretos en fechas únicas, descontextualizados de toda realidad cultural, social, política y económica, y por qué no ambiental. De ahí que surja el interrogante acerca de la posible articulación de la educación en ciencias con la historia y el contexto cultural.

El Esquema 1, intenta mostrar, que de una u otra forma las relaciones entre la historia, la cultura y la educación en ciencias deben ser tenidas en cuenta en los diferentes momentos de formación en esta área. Sin embargo, este apenas es un esbozo, ya que la intención de este trabajo es posibilitar socializaciones y discusiones en torno a cuales seria esas relaciones y como estas influyen directa o indirectamente o definitivamente no afectan los procesos de EyA de las ciencias.

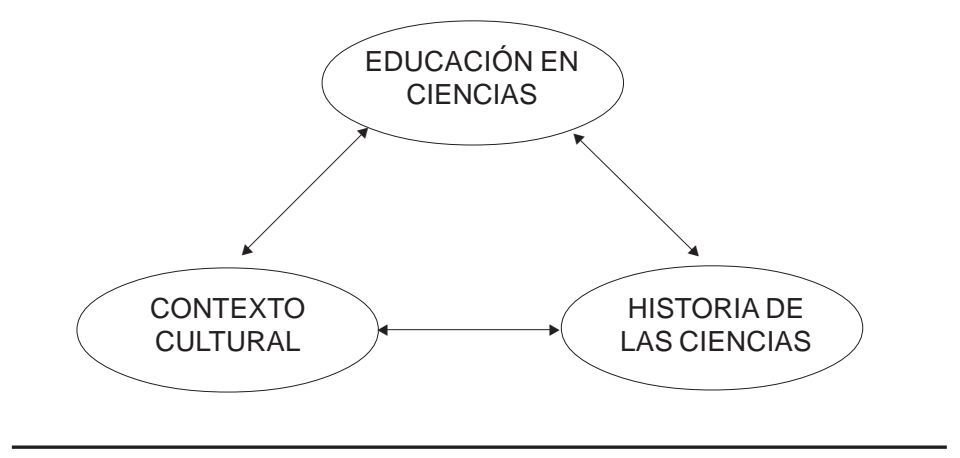

Esquema 1. Triangulación de los ejes temáticos (GARAY, 2007). 
Perspectivas de historia y contexto cultural ...

\section{Consideraciones finales}

Una educación en ciencias, desde una perspectiva histórica y cultural, permite mejorar los procesos de EyA, según resultados de investigaciones y de la formulación de nuevas propuestas metodológicas y didácticas. Esto es, de un lado, dotar de herramientas al docente para que pueda reflexionar sobre su que hacer en las aulas de formación, diseñando, formulando y reestructurando metodologías, procedimientos y estrategias de enseñanza, que permitan procesos de conceptualización más complejos, de otro lado, en los estudiantes, permite la contextualización de los saberes y practicas científicas en contextos únicos y específicos, delimitados por dimensiones políticas, económicas, sociales y culturales, permitiendo entre otras cosas, una reestructuración de la imagen de ciencia y de científicos, (humanización de las ciencias), así como una conceptualización en un nivel de complejidad mayor.

La reconstrucción de hechos históricos en ciencias, deben de una forma explícita contemplar los aspectos socioculturales, que podrán ser extrapolados a los contextos culturales de formación en ciencias, es decir, identificar dimensiones, valores, características generales y particulares de los contexto socio históricos relacionados con la formulación de saberes y prácticas científicas, que puedan ser extrapoladas a las aulas de formación y así, hacer de los procesos de humanización y transformación de la imagen de ciencia un verdadero proceso y no solo una intención.

La inclusión de la perspectiva socio cultural en los procesos de EyA de las ciencias, ha sido tenido en cuenta en trabajos e investigaciones que se han realizado en didáctica de las ciencias, particularmente en la reconstrucción de las hechos históricos, ya que esta perspectiva contempla dimensiones que son objeto de trabajo de otras áreas como son la sociología o la antropología de las ciencias, con ello entonces se pretende dejar en los lectores más que respuesta son muchos interrogantes en su que hacer como investigadores y como profesores de ciencias que nos ayuden a consolidar este enfoque como una nueva forma de educación en ciencias, que realmente no se limite a una simple homogenización de conceptos descontextualizados y sin formas de significación relevantes.

\section{Referências}

ADÚRIZ-BRAVO, A. Acerca de la didáctica de las ciencias como disciplina. Revista Electrónica de Enseñanza de las Ciencias, Vigo, v. 1, n. 3, p. 130-140, 2002.

ANDRADE, A. M. Conhecimento, cultura e escola: um estudo de suas inter-relações a partir das idéias dos alunos (8-12 anos) sobre os espinhos do cactus. 2000. 248f. Tese (Doutorado em Educação) - Faculdade de Educação, Universidade de São Paulo, São Paulo, 2000.

BACHELARD, G. La formación del espíritu científico. Mexico: Siglo Veintiuno, 1985.

BENSAUDE-VICENTE, B. Historia de las ciencias. In: SCERRI, M. (Ed.). Mendeleiev: historia de un descubrimiento. Madrid: Cátedra, 1991. p. 502-525. 
Garay, F. R. G.

FORQUIN, J.-C. Escola e cultura: as bases sociais e epistemológicas do conhecimento escolar. Porto Alegre: Ed. Artes, 1993.

GALAGOVSKI, L.; ADÚRIZ-BRAVO, A. Modelos y analogías en la enseñanza de las ciencias naturales: el concepto de modelo didáctico analógico. Enseñanza de las Ciencias, Barcelona, v. 19, n. 2, p. 231-242, 2001.

GARAY, F. Modelos abstractos en la formación inicial de profesores: constructos en la enseñanza/aprendizaje de periodicidad química. 2007. 214f. Dissertação (Mestrado em Educação) - Universidad Pedagogica Nacional, Bogotá, 2007.

GARAY, F; PÉREZ, R.; GALLEGO, R. Desarrollo histórico epistemológico del modelo de tabla periódica y periodicidad química. Tecné, Episteme y Didáxis, Bogotá, n. extra, p. 233-235, 2006.

GEERTZ, C. La interpretación de las culturas. Mexico: Gedisa, 1987.

IZQUIERDO, M. ¿Cómo contribuye la historia de las ciencias en las actitudes del alumnado hacia el aprendizaje de las ciencias? Aula de Innovación Educativa, La Rioja, n. 27 , p. 37-40, 1994.

KROEBER, L.; KLUCKHOHON, C. Cultura: una revisión critica de conceptos y definiciones. Papers of the Peabody Museum of American Archeology and Ethnology, Cambridge, v. 47, n. 1, 270-285, 1952.

MATTHEWS, M. Science teaching: the role of history and philosophy of science. New York: Routledge, 1994.

MENDELÉIEV, D. The principles of chemistry. NewYork: Prinston University, 1869.

MARTÍN DEL POZO, R.; SOLSONA, N. Los cambios químicos: de los modelos del alumnado a los modelos escolares. Alambique, Barcelona, n. 42, p. 19-28, 2004.

QUINTANILLA, M. Historia de la ciencia y formación del profesorado: una necesidad Irreducible. Tecné, Episteme y Didáxis, Bogotá, n. extra, p. 34-43, 2005.

SCERRI, E. The periodic table: its story and its significance. New York: Oxford University Press, 2007.

TOULMIN, S. Human understanding. Oxford: Clarendon Press, 1972.

YOUNG, M. Mental space. Online archive center for psychotherateutec studes, 1999. Disponível em: <http://www.human-nature.com/mental/chap3.html >. Acesso em: 28 mar. 2011.

Artigo recebido em maio de 2010 e aceito em outubro de 2010. 